\title{
Understanding human values and their emotional effect
}

\author{
Alexandra Balahur \\ European Commission Joint Research Centre \\ Directorate I, Unit I3 Text and Data Mining \\ Via E. Fermi 2749, 21027 Ispra (VA), Italy \\ alexandra.balahurdec.europa.eu
}

\begin{abstract}
1 Abstract

Emotions can be triggered by various factors. According to the Appraisal Theories (De Rivera, 1977; Frijda, 1986; Ortony et al., 1988; JohnsonLaird and Oatley, 1989) emotions are elicited and differentiated on the basis of the cognitive evaluation of the personal significance of a situa-tion, object or event based on appraisal criteria (intrinsic characteristics of objects and events, sig-nificance of events to individual needs and goals, individuals ability to cope with the con-sequences of the event, compatibility of event with social or personal standards, norms and val-ues). These differences in values can trigger re-actions such as anger, disgust (contempt), sad-ness, etc., because these behaviors are evaluated by the public as being incompatible with their social/personal standards, norms or values. Such arguments are frequently present both in main-stream media, as well as social media, building a society-wide view, attitude and emotional reac-tion towards refugees/immigrants. In this demo, I will talk about experiments to annotate and de-tect factual arguments that are linked to human needs/motivations from text and in consequence trigger emotion in the media audience and pro-pose a new task for next year's WASSA.
\end{abstract}

\section{References}

A. Ortony, G.L. Clore, and A. Collins, The Cognitive Structure of Emotions. Cambridge Univ. Press, 1988.

J. De Rivera, A Structural Theory of the Emotions, Psychological Issues, vol. 10, no. 4, 1977.

N. Frijda, The Emotions. Cambridge Univ. Press, 1986.
P.N. Johnson-Laird and K. Oatley, The Language of Emotions: An Analysis of a Semantic Field, Cognition and Emotion, vol. 3, pp. 81-123, 1989. 